\title{
RELAÇÃO ENTRE QUALIDADE DE VIDA, BURNOUT E CONDIÇÕES DE SAÚDE ENTRE BOMBEIROS MILITARES
}

\author{
Priscylla Ruany Mendes PESTANA ${ }^{1}$ \\ Talyta Emanuelle Ávila SILVA ${ }^{2}$ \\ Idael Emiliano Gomes SILVA ${ }^{3}$ \\ Danilo Lima CARREIRO ${ }^{4}$ \\ Laura Tatiany Mineiro COUTINHO \\ Wagner Luiz Mineiro COUTINHO
}

\begin{abstract}
1. Estudante do Curso de Graduação em Fisioterapia da Associação Educativa do Brasil (SOEBRAS), priscyllapestana@gmail.com

2. Estudante do Curso de Graduação em Fisioterapia da SOEBRAS, emanuelleavila10@yahoo.com.br

3. Pós-graduado em Fisioterapia Ortopédica e Postural (SOEBRAS). Professor da SOEBRAS,

idaelemiliano@yahoo.com.br

4. Mestre em Ciências da Saúde pela Universidade Estadual de Montes Claros (Unimontes). Professor das Faculdades

Santo Agostinho (FASA) e da SOEBRAS, danilolimacarreiro@gmail.com

5. Mestre em Ciências da Saúde pela Unimontes. Professora da SOEBRAS, mineiro.laura@gmail.com

6. Mestre em Ciências da Saúde pela Unimontes. Professor da SOEBRAS, coutinhowlm@ gmail.com
\end{abstract}

\section{Recebido em: 30/05/2014 - Aprovado em: 30/06/2014 - Disponibilizado em: 30/07/2014}

RESUMO: este estudo teve por objetivo determinar a relação entre percepção do nível de Qualidade de Vida, Síndrome de Burnout e condições de saúde entre bombeiros militares. Trata-se de um estudo transversal analítico no qual se utilizaram para coleta de dados: WHOQOL-Bref, Maslach Burnout Inventory, Critério de Classificação Econômica Brasil e Questionário demográfico-socioeconômico, condições laborais e de saúde. Através de regressão multivariada registrou-se maior chance de impacto negativo na percepção do nível de qualidade de vida geral entre bombeiros com indícios de desenvolvimento de SB quando comparados àqueles sem indícios de desenvolvimento desta síndrome. Maiores chances de baixas percepções tanto no nível de QV geral quanto no domínio psicológico foram identificadas entre pessoas do sexo feminino quando comparadas às do sexo masculino; entre bombeiros com indícios de desenvolvimento da dimensão exaustão emocional da SB, quando comparados àqueles sem indícios de desenvolvimento desta dimensão; entre bombeiros com Índice de Massa Corporal categorizado como sobrepeso/obeso, quando comparados àqueles com tal índice categorizado como normal; entre aqueles com níveis de pressão arterial sistólica categorizada como alta, quando comparados aos que apresentaram níveis de pressão arterial sistólica categorizada como normal. Os bombeiros apresentaram altos níveis de percepção de qualidade de vida geral e por domínios. Todavia, ao considerar os resultados registrados sugere-se a implementação e/ou revisão das medidas favoráveis à prevenção da Síndrome de Burnout, sobretudo de sua dimensão exaustão emocional, do sobrepeso/obesidade e da hipertensão arterial, principalmente entre bombeiros do sexo feminino.

Palavras-chave: Qualidade de vida. Esgotamento Profissional. Nível de Saúde. Bombeiros. Saúde do trabalhador.

\section{RELATIONSHIP BETWEEN QUALITY OF LIFE, BURNOUT AND HEALTH AMONG FIREFIGHTERS}

\begin{abstract}
Quality of Life (QL), Burnout Syndrome (SB) and health conditions among firefighters. This is an analytical cross-sectional study in which it was used for data collection: WHOQOL - Bref, Maslach Burnout Inventory, Critério de Classificação Econômica Brasil and socioeconomic-demographic, labor and health conditions questionnaire. Through logistic regression was registered greater chance of negative impact on the perceived level of overall quality of life among firefighters with evidence of development of SB compared to those without evidence of development of this syndrome. Higher chances of low-level perceptions of overall QL as in the psychological domain were identified among females compared to males; among firefighters with evidence for the development of emotional exhaustion dimension of SB compared to those without evidence of developing this dimension; among firefighters with body mass index categorized as overweight/obese, compared to those with such index categorized as normal; among those with levels of systolic blood pressure categorized as high, compared with those who had higher levels of systolic blood pressure categorized as normal. Firefighters had high levels of perceived overall quality of life and fields. However, when considering the recorded results suggest the implementation and/or revision of favorable prevention of burnout syndrome measures, particularly their emotional exhaustion dimension, overweight/obesity and hypertension, especially among female firefighters.
\end{abstract}

Keywords: Quality of Life. Burnout, Professional. Health Status. Firemen. Occupational Health. 


\section{INTRODUÇÃO}

Universalmente, o termo Qualidade de vida (QV) envolve significados diversos fundamentados no conhecimento, na experiência e nos valores das pessoas ao considerar sua satisfação material em relação às necessidades elementares da vida humana (alimentação, saneamento, habitação, educação, saúde, trabalho e lazer) bem como em relação aos valores não materiais (amor, liberdade, solidariedade, inserção social, realização pessoal e felicidade). $\mathrm{Na}$ área da saúde, a relação entre QV, condições de saúde e saúde teve sua maior expressão a partir dos séculos XVIII e XIX com o advento da medicina social, a qual passou a fomentar políticas públicas e movimentos sociais tendo como horizonte norteador a promoção da saúde. (MINAYO, 2000).

Neste contexto, as pessoas têm na profissão e no trabalho a possibilidade de realização de suas satisfações, sendo que a partir das atividades laborais a pessoa não somente transforma e cria coisas, mas também se transforma e recria-se. Todavia, a profissão e o trabalho, além de produzirem bem-estar físico e emocional, podem ainda favorecer o desenvolvimento de insatisfações, sofrimentos e estresse no trabalhador. (DEJOURS, ABDOUCHELI e JAYET, 1994; MINAYO, ASSIS, OLIVEIRA, 2011).

Entre as categorias profissionais, a do bombeiro militar (BM) tem um contexto laboral diversificado, o qual compreende o encargo de preservar a vida, o meio ambiente, o patrimônio e a ordem pública; atuar em prevenção e extinção de incêndios, em proteção e salvamento e em emergências préhospitalares de vítimas de afogamentos, inundações, desabamentos, acidentes em geral, catástrofes e calamidades públicas. (GONZALES et al., 2006; NATIVADE, 2009; ZACONETA et al., 2010). De acordo com a epidemiologia das doenças do trabalho, tal contexto permeia probabilidades, exposições e situações ou fatores de risco que podem determinar efeitos adversos no processo de trabalho, saúde-doença. (MACHADO, 1997). Para militares, há de considerar ainda que o conceito de situações ou fatores de risco extrapola o dispositivo técnico por constituir um conceito estruturante da própria atividade profissional. (MINAYO, ASSIS, OLIVEIRA, 2011).

Como outros profissionais que exercem atividades laborais associadas à constante pressão emocional e exigência do estado de prontidão, às situações de emergências rotineiras e repetitivas, às escalas desgastantes, aos contatos interpessoais intensos e ao atendimento pré-hospitalar, os bombeiros militares (BM) podem ser acometidos por estresse crônico -Síndrome de Burnout (SB) .(GONZALES et al., 2006; SERRRALHEIRO et al., 2011; FRANCA et al., 2012). Como resposta fisiológica ao estresse crônico pode ocorrer estímulo do Sistema Nervoso Simpático (SNS) e do 
Sistema Neuroendócrino desencadeando quadros de hipertensão arterial sistêmica (HAS) e de doenças cardiovasculares (DC). (DIAZ-RODRIGUEZ, 2011).

Desta forma este estudo teve por objetivo determinar a relação entre percepção do nível de qualidade de vida e condições de saúde entre bombeiros militares.

\section{METODOLOGIA}

Estudo transversal censitário entre militares de um batalhão do corpo de bombeiros de uma cidade de grande porte no estado de MG. A coleta dos dados ocorreu entre maio e junho de 2012, por meio de entrevistas estruturadas, avaliação antropométrica e dos níveis pressóricos, por pesquisadores examinadores previamente treinados e calibrados. O período matutino, preferencialmente antes do início das atividades ocupacionais, foi priorizado para a coleta de dados, com o intuito de melhor controle da qualidade da antropometria e dos níveis pressóricos.

Considerou-se como variável dependente, a percepção do nível de QV, avaliada pelo WHOQOL-bref. Trata-se de um questionário validado para uso no Brasil, constituído por 26 itens que envolvem os domínios físico, psicológico, social e ambiental, no qual maior escore corresponde à melhor percepção do nível de QV. (FLECK et al., 2000). As variáveis independentes foram classificadas em variáveis individuais (demográficas, socioeconômicas e de saúde) e variáveis ocupacionais.

$\mathrm{Na}$ Tabela 1 são apresentadas as categorizações das variáveis em estudo.

Tabela 1 - Categorizações das variáveis, relação entre qualidade de vida, Burnout e condições de saúde entre bombeiros militares do interior de MG, $2012(\mathrm{n}=202)$

\begin{tabular}{|c|c|c|}
\hline Variáveis & Categorização & Referência categorização \\
\hline \multicolumn{3}{|l|}{ Dependentes } \\
\hline Percepção nível QV geral ${ }^{\mathrm{a}}$ & $0=$ alta $1=$ baixa & limite inferior intervalo de confiança \\
\hline Percepção nível QV domínio físico ${ }^{\mathrm{a}}$ & $0=$ alta $1=$ baixa & limite inferior intervalo de confiança \\
\hline Percepção nível QV domínio psicológico ${ }^{\mathrm{a}}$ & $0=$ alta $1=$ baixa & limite inferior intervalo de confiança \\
\hline Percepção nível QV domínio social ${ }^{\mathrm{a}}$ & $0=$ alta $1=$ baixa & limite inferior intervalo de confiança \\
\hline Percepção nível QV domínio ambiental $^{\mathrm{a}}$ & $0=$ alta $1=$ baixa & limite inferior intervalo de confiança \\
\hline \multicolumn{3}{|l|}{ Independentes } \\
\hline \multicolumn{3}{|l|}{ Individuais } \\
\hline \multicolumn{3}{|l|}{ Demográficas } \\
\hline Sexo & $0=$ masculino $1=$ feminino & b \\
\hline Idade & $0=>27$ anos e $1=\leq 27$ anos & menor quartil \\
\hline Raça ou cor da pele ${ }^{c}$ & $0=$ branca $/$ amarela $1=$ preta $/$ parda/indígena & - \\
\hline \multicolumn{3}{|l|}{ Socioeconômicas } \\
\hline Estado civil ${ }^{\mathrm{c}}$ & $0=$ casado $1=$ solteiro $/$ divorciado $/$ desquitado $^{\mathrm{d}}$ & b \\
\hline Segmentação econômica ${ }^{\mathrm{e}}$ & $0=\operatorname{alta}(\mathrm{A} 1 / \mathrm{B} 2) 1=$ baixa $(\mathrm{C} 1 / \mathrm{E})$ & f \\
\hline Escolaridade & $0=$ ensino superior $1=$ ensino médio & - \\
\hline \multicolumn{3}{|l|}{ Condições de saúde } \\
\hline Síndrome de Burnout & $0=$ ausência $1=$ presença & - \\
\hline Exaustão emocional & $0=$ baixa $/$ moderada $1=$ alta & - \\
\hline Despersonalização & $0=$ baixa $/$ moderada $1=$ alta & - \\
\hline Realização profissional & $0=$ baixa $/$ moderada $1=$ alta & - \\
\hline Tabagismo & $0=$ não $1=\operatorname{sim}$ & \\
\hline Consumo de bebidas alcóolicas & $0=$ não $1=\operatorname{sim}$ & \\
\hline
\end{tabular}


Estado nutricional (IMC) $)^{\mathrm{g}_{*}}$

Índice de Conicidade (IC) ${ }^{\mathrm{h}}$

Circunferência Abdominal (CA) ${ }^{\mathrm{i}}$

Relação Cintura Quadril (RCQ) ${ }^{\mathrm{j}}$

Razão Cintura Estatura (RCEst) ${ }^{\mathrm{k}}$

Pressão arterial sistólica e diastólica ${ }^{1}$

Ocupacionais

Posse de outro emprego

Estrutura organizacional

Tempo de serviço
$0=$ normal $1=$ sobrepeso/obeso

$0=$ sem risco $1=$ com risco

$0=$ sem risco $1=$ com risco

$0=$ sem risco $1=$ com risco

$0=$ sem risco $1=$ com risco

$0=$ não e $1=\operatorname{sim}$

$0=$ não $1=\operatorname{sim}$

$0=$ administrativo e $1=$ operacional

$0=>14$ anos; $1=>$ sete $\mathrm{e} \leq 14 ; 2=>$ três $\mathrm{e} \leq$ quartis sete anos; $3=\leq$ três anos

\section{${ }^{\mathrm{a}}$ Investigada de acordo com o Whoqol-Bref.}

${ }^{\mathrm{b}}$ BLAY E MARCHESONI (2011).

'Investigada de acordo com o "Questionário da Amostra CD 2010”, proposto pelo Instituto Brasileiro de Geografia e Estatística (IBGE).

d،casado" compreendeu os sujeitos casados e com união estável, e "solteiro/ divorciado" os desquitados ou separados judicialmente, os divorciados, os viúvos e os solteiros.

eInvestigada de acordo com o Critério de Classificação Econômica Brasil (CCEB). Tais segmentações representam as respectivas classes de renda familiar: A1: R\$ 12.926,00; A2: R\$ 8.418,00; B1: R\$ 4.418,00; B2: R\$ 2.565,00; C1: R\$ 1.541,00; C2: R\$ 1.024,00; D: R\$ 714,00 e E: R\$ 477,00.

${ }^{\mathrm{f}}$ RECHENCHOSKY et al. (2009).

${ }^{\mathrm{g}}$ Calculado pela razão peso/altura ${ }^{2}\left(\mathrm{~kg} / \mathrm{m}^{2}\right)$, e posteriormente classificado de acordo com a OMS em índice normal $\left(<25 \mathrm{~kg} / \mathrm{m}^{2}\right)$; sobrepeso $\left(25\right.$ a $\left.29,9 \mathrm{~kg} / \mathrm{m}^{2}\right)$ ou obeso $\left(\geq 30 \mathrm{~kg} / \mathrm{m}^{2}\right)($ CIPULLO et al. 2010).

${ }^{\mathrm{h}}$ Considerou como discriminador de risco elevado os pontos de corte: 1,25 para homens e 1,18 para mulheres (PITANGA E LESSA, 2011).

${ }^{\mathrm{i}}$ Considerou CA $>80 \mathrm{~cm}$ e $>94 ; \mathrm{CA}>88 \mathrm{~cm}$ e $>102$, respectivamente para mulheres e homens, como risco aumentado e risco muito elevado que constituíram uma única categoria (LEAN, HAN e MORRISON, 1995).

${ }^{\mathrm{j}}$ Considerou como ponto de corte respectivamente RCQ $\leq 0,85$ e RCQ $\leq 1,0$ para mulheres e homens (GOSTON e MENDES, 2001).

${ }^{\mathrm{k}}$ Considerou como ponto de corte o valor $\geq 0,5$ para ambos os gêneros (HSIEH e MUTO, 2005).

${ }^{\mathrm{l}}$ Realizada com o indivíduo sentado, após 5 minutos de descanso, com a bexiga vazia, e após pelo menos 30 minutos sem ingestão de alimentos, cafeína e/ou consumo de cigarros. Foram realizadas três medidas, desprezando-se a primeira. A pressão arterial foi considerada como a média aritmética da segunda e terceira medidas (BORIM, GUARINETO e ALMEIDA, 2011). Os pontos de corte considerados para pressão alta consideraram os valores preconizados pelo Joint Committee on Detection, Evaluation and Treatment of High Blood Pressure, que estipula valor $\geq$ a $140 \mathrm{~mm} / \mathrm{Hg}$ para pressão sistólica alta e $\geq$ a $90 \mathrm{~mm} / \mathrm{Hg}$ para pressão diastólica alta (JVC VII, 2003) e/ou em uso de drogas anti-hipertensivas (SCHEFFEL et al., 2004).

*IMC: índice de massa corporal.

Para avaliação antropométrica e dos níveis pressóricos foram utilizados os seguintes instrumentos: fita métrica inextensível retrátil Fiber Glass ${ }^{\circledR}$, balança mecânica antropométrica Filizola ${ }^{\circledR}$, estetoscópios Rappaport Premium ${ }^{\circledR}$ e esfigmomanômetros Aneróide Premium ${ }^{\circledR}$, calibrados e certificados pelo Instituto Nacional de Metrologia, Normalização e Qualidade Industrial. (INMETRO).

A análise estatística se deu pelo software Statistical Package for the Social Sciences - SPSS v.17.0. Para análise do teste/reteste, a concordância entre examinadores foi calculada pelo coeficiente
Kappa de Cohen $(\mathrm{K})$ para variáveis nominais conforme concordância: desprezível $(<0,20)$; mínima $(0,20-0,40)$; ruim $(0,41-0,60)$; boa $(0,61-0,80) ; \quad$ excelente $\quad(0,81-1,00)$. (MASCARENHAS e GOMES, 2011). A análise da confiabilidade inter observador dos indicadores antropométricos e dos níveis pressóricos (variáveis quantitativas) deu-se pelo Coeficiente de Correlação Intra Classe $(\mathrm{CCI}): \quad<0,70$ não aceitáveis; $\geq 0,7$ suficientemente reprodutível; $0,71-0,79$ aceitáveis; 0,80- 0,89 muito bons e $\geq 0,90$ excelentes. (SOUZA et a., 2011).

Para avaliar associação entre percepção do nível de QV e variáveis 
independentes, realizou-se análise bivariada através do Teste Qui-quadrado considerando associação ao nível de $\mathrm{p} \leq 0,20$. Variáveis independentes que se mostraram associadas foram incluídas nas análises de regressão logística múltipla retendo no modelo final aquelas que se associaram com QV ao nível de $\mathrm{p} \leq 0,05$.

Estudo aprovado pelo Comitê de Ética da Associação Educativa do Brasil (SOEBRAS) com parecer consubstanciado $\mathrm{n}^{\circ}$ 19893.

\section{RESULTADOS E DISCUSSÃO}

Identificaram-se na coleta de dados 275 bombeiros militares lotados no batalhão. Desses, 48 foram considerados inelegíveis: 13 participavam de cursos em outra cidade e 35 estavam de férias. Dos 227 trabalhadores considerados elegíveis, 202 consentiram participar do estudo (taxa de resposta de 88,99\%). As análises do teste $\mathrm{K}$ e CCI evidenciaram consistência inter e intra observadores. Os bombeiros apresentaram média de idade de 32,15 anos $\left( \pm 7,12 ; \mathrm{IC}_{95 \%}\right.$ : $31,16-33,14 ;$ 21-50) e tempo de serviço médio: 9,39 anos ( $\pm 7,51$; $\mathrm{IC}_{95 \%}$ : 8,34-10,42; 1,5-29). A avaliação da percepção do nível de QV geral e por domínios (físico, psicológico, social e ambiental) identificou altos níveis de percepção entre $78,2 \% \quad(n=158) ; \quad 66,8 \%$ $(\mathrm{n}=135) ; \quad 59,4 \% \quad(\mathrm{n}=120) ; 47,5 \% \quad(\mathrm{n}=96) ;$ $57,4 \%(n=116)$ respectivamente. Nas bases de dados consultadas não se identificaram estudos prévios acerca de fatores relacionados à percepção do nível de QV entre bombeiros que melhor subsidiariam a discussão dos resultados do presente estudo. Entre policiais militares das cinco grandes regiões do estado de Santa Catarina registrou que as médias da percepção dos níveis de QV geral e por domínios (físico, psicológico, social e ambiental) foram respectivamente de: 61,4; 54,1; 62,5; 75,0; 53,1. (SILVA et al., 2012).

Quanto às variáveis independentes que se mostraram associadas à percepção do baixo nível de QV e foram incluídas nas análises de regressão logística múltipla registou-se que 9,4\% ( $\mathrm{n}=19)$ era do sexo feminino; $55,9 \%$ $(\mathrm{n}=113)$ apresentou indícios de desenvolvimento da SB; 20,3\% (n=41) exibiu indícios de desenvolvimento da dimensão exaustão emocional da SB; 53,9\% (n=109) teve o estado nutricional categorizado como sobrepeso/obeso; e 11,9\% ( $n=24)$ apresentou nível de pressão arterial sistólica alta.

$\mathrm{Na}$ Tabela 2 são apresentados os resultados do modelo final. Registrou-se maior chance de baixa percepção no nível de QV geral entre bombeiros com indícios de desenvolvimento da SB quando comparados àqueles sem indícios de desenvolvimento desta síndrome.

Maiores chances de baixa percepção tanto no nível de QV geral quanto no domínio psicológico foram identificadas entre pessoas do sexo feminino quando comparadas às do sexo masculino; entre bombeiros com indícios 
de desenvolvimento da dimensão exaustão emocional da SB, quando comparados àqueles sem indícios de desenvolvimento desta dimensão; entre bombeiros com IMC categorizado como sobrepeso/obeso, quando comparados àqueles com IMC categorizado como normal; entre aqueles com níveis de pressão arterial sistólica categorizada como alta, quando comparados aos que apresentaram níveis de pressão arterial sistólica categorizada como normal.

Tabela 2 - Análise de regressão logística múltipla da relação entre qualidade de vida, Burnout e condições de saúde entre bombeiros militares do interior de MG, 2012 ( $n=202)$

\begin{tabular}{|c|c|c|c|c|c|c|}
\hline & \multicolumn{3}{|c|}{ QV Geral } & \multicolumn{3}{|c|}{ QV Domínio Psicológico } \\
\hline VARIÁVEIS & OR & $\mathrm{IC}_{95 \%}$ & $p$ & OR & $\mathrm{IC}_{95 \%}$ & $p$ \\
\hline \multicolumn{7}{|l|}{ Demográficas } \\
\hline \multicolumn{7}{|l|}{ Sexo } \\
\hline Masculino & 1,00 & & & 1,00 & & \\
\hline Feminino & 3,79 & $1,16-12,32$ & 0,027 & 4,28 & $1,53-12,02$ & 0,006 \\
\hline \multicolumn{7}{|l|}{ Condições de saúde } \\
\hline \multicolumn{7}{|c|}{ Síndrome de Burnout } \\
\hline Ausência & 1,00 & & & -- & -- & -- \\
\hline Presença & 3,55 & $1,33-9,46$ & 0,01 & -- & -- & -- \\
\hline \multicolumn{7}{|l|}{ Exaustão emocional } \\
\hline Ausência & 1,00 & & & 1,00 & & \\
\hline Presença & 4,82 & $1,96-11,83$ & 0,001 & 7,70 & $3,39-17,50$ & 0,000 \\
\hline \multicolumn{7}{|c|}{ Estado nutricional (IMC) } \\
\hline Normal & 1,00 & & & 1,00 & & \\
\hline Sobrepeso/obeso & 2,14 & $1,09-4,70$ & 0,049 & 2,17 & $1,13-4,16$ & 0,020 \\
\hline \multicolumn{7}{|c|}{ Pressão arterial sistólica } \\
\hline Normotenso & 1,00 & & & 1,00 & & \\
\hline Hipertenso & 2,91 & $1,01-8,38$ & 0,048 & 2,51 & $1,02-6,48$ & 0,050 \\
\hline
\end{tabular}

Profissionais do sexo feminino apresentaram maior chance de baixa percepção tanto no nível de QV geral quanto no domínio psicológico que compreende sentimentos positivos, pensamento, aprendizagem, memória e concentração, autoestima, imagem corporal e aparência e sentimentos negativos. (TABELEÃO, TOMASI, NEVES, 2011). Estudo prévio identificou associação entre sexo feminino e domínio psicológico possivelmente justificado pela maior disponibilidade das mulheres em abordar questões subjetivas bem como maior facilidade em expressar experiências interiores. (BLAY E MARCHESONI, 2011). Ressalta-se ainda que geralmente as mulheres tenham jornada de trabalho formal e informal, o pode contribuir para percepção negativa da QV. (OLIVEIRA et al., 2012).

Constatou-se maior chance de baixa percepção no nível de QV geral com indícios de desenvolvimento da SB. Para avaliar QV geral por meio do WHOQOL-bref, consideram-se as duas primeiras questões ("como você avaliaria sua qualidade de vida?" e "quão satisfeito(a) você está com a sua saúde?”). Estas são calculadas em conjunto 
para gerar um único escore independente dos escores dos domínios. (MICHELONE e SANTOS, 2004). Quanto á SB, esta é característica do meio laboral e tida como um processo que se dá em resposta à cronificação do estresse, com possíveis consequências negativas tanto em nível individual, como profissional, familiar e social. (GUIDO et al., 2012). Desta forma, acredita-se que a relação identificada possa justificar-se na exposição destes trabalhadores a agentes ocupacionais estressores comuns à profissionais de urgência pré-hospitalar como ambiente insalubre, escalas de trabalho por turnos e intenso contato com os usuários do serviço demarcado por fortes emoções, (RIOS, 2008; FERNANDES et al., 2012), que combinados às peculiaridades biológicas da pessoa podem resultar em doenças e agravos (MINAYO, ASSIS e OLIVEIRA, 2011) impactando assim negativamente na satisfação do trabalhador com seu estado de saúde.

A QV geral e o domínio psicológico relacionaram-se à dimensão exaustão emocional da SB. Resultado convergente à estudo entre professores de Pelotas (RS) o qual também identificou relação entre domínio psicológico e exaustão emocional. (TABELEÃO, TOMASI, NEVES, 2011). Tal dimensão caracteriza-se pelo esgotamento emocional decorrente da sobrecarga e do conflito pessoal nas relações interpessoais. (PEREIRA, 2002). Desta forma, ao se considerar o contexto laboral do bombeiro, demarcado por situações inesperadas, bem como pelo contingente de atividades a serem desempenhadas, as quais exigem alto nível em sua execução, este profissional acaba submetido a cargas psíquicas e físicas. (GONZALES et al., 2006). O conflito pessoal experimentado por tal profissional por sua vez, é inerente a trabalhadores assistencialistas, uma vez que ao mesmo tempo em que necessitam estabelecer vínculos afetivos para prestar o cuidado, têm que romper tais vínculos face o contexto profissional ser mediado por normas, horários, transferências e com a própria morte. (JACQUES, 2004).

Bombeiros com sobrepeso/obesidade também tiveram maior chance de impacto negativo na percepção da QV geral e no domínio psicológico. Estudos prévios identificaram associação entre piores escores de QV e obesidade. (BACKES et al., 2011; OLIVEIRA-CAMPOS et al., 2013). Tais resultados vão de encontro à maioria das pesquisas prévias que identificaram maior comprometimento da saúde mental e física entre obesos. (SILVA et al., 2006). Entre militares, estudos prévios têm identificado medidas de peso corporal elevados com prevalência de 19,5\% entre cariocas. (MINAYO, ASSIS, OLIVEIRA, 2011). Entre bombeiros da cidade de Pelotas (RS) o escore médio de IMC foi de $26,0 \mathrm{Kg} / \mathrm{m}^{2}$, caracterizado sobrepeso. (CANABARRO e ROMBALDI, 2010). O impacto no domínio 
psicológico pode ser resultado da percepção da imagem corporal e aparência. Neste sentido estudos têm identificado que pessoas com excesso de peso podem apresentar limitações emocionais e físicas; (BACKES et al., 2011); prejuízo do bem-estar emocional e do status funcional, (RICHARDS, ADAMS e HUNT, 2000), além de exposição à situações humilhantes em decorrência de suas restrições físicas. (WADDEN e STUNKARD, 1985).

As percepções do nível de QV geral e do domínio psicológico foram ainda impactadas negativamente pela hipertensão arterial sistólica. Estudo desenvolvido entre hipertensos de Fortaleza (CE) identificou que a cronicidade da hipertensão pode impactar negativamente no estado geral de saúde e na percepção da QV entre todos os domínios do Medical Outcomes Study 36-Item Short-Form Health Survey (SF-36). (BRITO et al., 2008). Outras pesquisas identificaram que os principais fatores que impactam na $\mathrm{QV}$ dos hipertensos são as complicações da hipertensão arterial e efeitos adversos das drogas anti-hipertensivas. (YOUSSEF, MOUBARAK e KAMEL, 2005). A possiblidade de complicações pode ainda abalar a autoestima do hipertenso e assim propiciar o comprometimento emocional pelo acometimento de transtornos depressivos e/ou ansiosos. (CURTIN e LUBKIN, 1990).

Destaca-se que a avaliação da percepção do nível de QV e das variáveis investigadas é dinâmico. Portanto, causas e efeitos certamente variam ao longo do tempo e, sendo este um estudo seccional, não é possível estabelecer uma relação temporal entre as associações observadas. Todavia, destaca-se que os resultados apresentados são confiáveis, frutos de um estudo censitário.

\section{CONCLUSÃO}

Os bombeiros apresentaram altos níveis de percepção de QV geral e por domínios. Todavia, registrou-se maior chance de impacto negativo na percepção do nível de qualidade de vida geral entre bombeiros com indícios de desenvolvimento de SB. Maiores chances de baixas percepções tanto no nível de QV geral quanto no domínio psicológico entre pessoas do sexo feminino; entre bombeiros com indícios de desenvolvimento da dimensão exaustão emocional da SB; entre bombeiros com Índice de Massa Corporal categorizado como sobrepeso/obeso; entre aqueles com níveis de pressão arterial sistólica categorizada como alta.

\section{REFERÊNCIAS}

BACKES, V. et al. Associação entre aspectos psicossociais e excesso de peso referido em adultos de um município de porte médio do sul do Brasil. Caderno de Saúde Pública, Rio de Janeiro, v. 27, n. 3, p. 573-580, 2011.

BLAY, S. L. MARCHESONI, M. S. M. Associação entre as condições físicas, psiquiátricas e socioeconômicos e pontuações WHOQOL-Bref. Cadernos de Saúde Pública, v. 27, n. 4, p. 677-686, 2011. 
BORIM, F. S. A. GUARIENTO, M. E. ALMEIDA, E. R. Perfil de adultos e idosos hipertensos em unidade básica de saúde.

Revista Brasileira de Clínica Médica, Botucatu, v. 9, p. 107-111, 2011.

BRITO, D. M. S. et al. Qualidade de vida e percepção da doença entre portadores de hipertensão arterial. Cadernos de Saúde Pública, Rio de Janeiro, v. 24, n. 4, p. 933940, 2008.

CANABARRO, L. K.ROMBALDI, A. J. Risco de sobrepeso e obesidade em soldados do corpo de bombeiros. Pensar a Prática, Goiânia, v. 13, n. 3, p. 1-13, 2010.

CIPULLO, J. P. et al. Prevalência e fatores de risco para hipertensão em uma população urbana brasileira. Arquivos Brasileiros de Cardiologia, Rio de Janeiro, v. 94, p. 519526, 2010.

CURTIN, M. LUBKIN, I. Chronic illness: impact and interventions. 2nd ed. Boston London: Jones and Bartlett; 1990.

DEJOURS, C. ABDOUCHELI, E. JAYET, C. Psicodinâmica do trabalho: contribuições da escola dejouriana à análise da relação prazer, sofrimento e trabalho. São Paulo: Atlas; 1994.

DIAZ-RODRIGUEZ, L. et al . Uma sessão de Reiki em enfermeiras diagnosticadas com síndrome de Burnout tem efeitos benéficos sobre a concentração de IgA salivar e a pressão arterial. Revista Latino-Americana de Enfermagem, Ribeirão Preto, v. 19, n. 5, p.1132-1138, 2011.

FERNANDES, A. $M$ et al. Síndrome de burnout em profissionais de enfermagem do serviço de atendimento móvel de urgência. Revista de Pesquisa: Cuidado é Fundamental Online, v. 4, n. 4, p. 31253135, 2012.

FLECK, M. P.A. et al . Aplicação da versão em português do instrumento abreviado de avaliação da qualidade de vida "WHOQOL- bref". Revista de Saúde Pública, São Paulo, v. 34, n. 2, p. 178-183, 2000.

FRANCA, S. P. S. et al. Preditores da síndrome de burnout em enfermeiros de serviços de urgência pré-hospitalar. Acta Paulista de Enfermagem, São Paulo, v. 25, n. 1, p. 68-73, 2012.

GONZALES, R. M. B. et al. O estado de alerta: um estudo exploratório com o corpo de bombeiros. Escola Anna Nery, Rio de Janeiro, v. 10, n. 3, p. 370-377, 2006.

GOSTON, J. L. MENDES, L. L. Perfil nutricional de praticantes de corrida de rua de um clube esportivo da cidade de Belo Horizonte, MG, Brasil. Revista Brasileira de Medicina do Esporte, São Paulo, v. 17, p. 13-17, 2011.

GUIDO LA et al. Síndrome de Burnout em residentes multiprofissionais de uma universidade pública. Revista da Escola de Enfermagem da USP, São Paulo, v. 46, n. 6, p. 1477-1483, 2012.

HSIEH, S. D. MUTO, T. The superiority of waist-to-height ratio as an anthropometric index to evaluate clustering of coronary risk factors among non-obese men and women. Preventive Medicine, Montreal, v. 40, p. 216-220, 2005.

JACQUES, M. G. Psicologia e trabalho. In: Guimarães, L. B. M. Ergonomia: tópicos especiais, qualidade de vida no trabalho, psicologia e trabalho. Porto Alegre: FEENG/ UFRGS/EE/PPGEP; 2004, p.1-14.

JVC VII. Seventh Report of the Joint National Committee on Prevention, Detection, Evaluation, and Treatment of High Blood Pressure. Journal of the American Medical Association, v. 289, p. 2560-2572, 2003.

LEAN, M. E. J. HAN, T. S. MORRISON, C. E. Waist circumference as a measure for indicating need for weight management. British Medical Journal, Londres, v. 311, p. 158-161, 1995. 
MACHADO, I. M. H. Processo de vigilância em saúde do trabalhador. Caderno de Saúde Publica, São Paulo, v. 13, supl. 2, p. 33,45, 1997.

MASCARENHAS, M. D. M. GOMES, K.R.O. Confiabilidade dos dados do Sistema de Informações sobre Nascidos Vivos em Teresina, Estado do Piauí, Brasil - 2002. Ciência e Saúde Coletiva, Rio de Janeiro, v. 16, p.1233-1239, 2011.

MICHELONE, A. P. C. SANTOS, V. L. C. G. Qualidade de vida de adultos com câncer colorretal com e sem ostomia. Revista Latino-Americana de Enfermagem, Ribeirão Preto, v. 12, n. 6, p. 875-83, 2004.

MINAYO, M. C. Qualidade de vida e saúde: um debate necessário. Ciência \& Saúde Coletiva, Rio de Janeiro, v. 5, n. 1, p. 7-18, 2000.

MINAYO, M. C. S. ASSIS, S. G. OLIVEIRA R. V. C. Impacto das atividades profissionais na saúde física e mental dos policiais civis e militares do Rio de Janeiro (RJ, Brasil). Ciência e Saúde Coletiva, Rio de Janeiro, v. 16, n, 4, p. 21992209, 2011.

NATIVADE, M. R. Vidas em risco: a identidade profissional dos bombeiros militares. Psicologia \& Sociedade, Belo Horizonte, v. 21, n. 3, p. 411-420, 2009.

OLIVEIRA-CAMPOS, M. et al. Impacto dos fatores de risco para doenças crônicas não transmissíveis na qualidade de vida. Ciência e Saúde Coletiva, Rio de Janeiro, v. 18, n. 3, p. 873-882, 2013.

OLIVEIRA, E. R. A. et al. Gênero e qualidade de vida percebida: estudo com professores da área de saúde. Ciência e Saúde Coletiva, Rio de Janeiro, v. 17, n. 3, p. 741-747, 2012.
PEREIRA, A. M. T. B. Burnout: quando o trabalho ameaça o bem-estar do trabalhador. São Paulo: Casa do Psicólogo; 2002.

PITANGA, F. J. G. LESSA, I. Sensibilidade e especificidade do índice de conicidade como discriminador do risco coronariano de adultos em Salvador, Brasil. Revista Brasileira de Epidemiologia, São Paulo, v. 7, p. 259-269, 2011.

RECHENCHOSKY, L et al. Estado nutricional e perfil lipídico de crianças. Revista da Educação Física/UEM, Maringá, v. 20, p. 431-440, 2009.

RICHARDS, M. M. ADAMS, T. D. HUNT, S. C. Functional status and emotional wellbeing, dietary intake, and physical activity of severely obese subjects. Journal of the American Dietetic Association, v. 100, p. 67-75, 2000.

RIOS, I. C. Humanização e ambiente de trabalho na visão de profissionais da saúde. Saúde e Sociedade, São Paulo, v. 17, n. 4, p. 151-160, 2008.

SCHEFFEL, R. S. et al. Prevalência de complicações micro e macrovasculares e de seus fatores de risco em pacientes com diabetes melito do tipo $2 \mathrm{em}$ atendimento ambulatorial. Revista da Associação Medica Brasileira, São Paulo, v. 50, p. 263-267, 2004.

SERRRALHEIRO, F. C. et al. Prevalência da síndrome de Burnout em anestesiologistas de Instituição de Ensino Superior em Medicina. Arquivos Brasileiros Ciências da Saúde, v.36, n. 3, p. 140-3, 2011.

SILVA, M. P. et al. Obesidade e qualidade de vida. Acta Médica Portuguesa, v. 19, p. 247250, 2006.

SILVA, R. et al. Aspetos relacionados à qualidade de vida e atividade física de policiais militares de Santa Catarina - Brasil. Motricidade, Vila Real, v. 8, n. 3, p. 81-89, 2012. 
SOUZA JA, et al. Biofotogrametria confiabilidade das medidas do protocolo do software para avaliação postural (SAPO).

Revista Brasileira de Cineantropometria e Desempenho Humano, Florianópolis, v. 13, p. 299-305, 2011.

TABELEÃO, V. P. TOMASI, E. NEVES, S. F. Qualidade de vida e esgotamento profissional entre docentes da rede pública de Ensino Médio e Fundamental no Sul do Brasil. Cadernos de Saúde Pública, Rio de Janeiro, v. 27, n. 12, p. 2401-2408, 2011.

YOUSSEF, R. M. MOUBARAK, II KAMEL, M.I. Factors affecting the quality of life of hypertensive patients. Eastern

Mediterranean Health Journal, v. 11, v. 12, p. 109-118, 2005.

WADDEN, T. A. STUNKARD, A. J. Social and psychological consequences of obesity. Annals of Internal Medicine, v. 103, p. 1062-1067, 1985.

ZACONETA CAM et al . Avaliação do pico de pressão e da frequência respiratória durante o uso de balão autoinflável por socorristas do Corpo de Bombeiros em um modelo de pulmão neonatal pré termo. Revista Paulista de Pediatria, São Paulo, v. 28, n. 1, p. 5-9, 2010. 